Proyecciones Journal of Mathematics

Vol. 29, No 2, pp. 101-108, August 2010.

Universidad Católica del Norte

Antofagasta - Chile

DOI: 10.4067/S0716-09172010000200003

\title{
JEWELL THEOREM FOR HIGHER DERIVATIONS ON $C^{*}$-ALGEBRAS
}

\author{
SHIRIN HEJAZIAN \\ MADJID MIRZAVAZIRI \\ and \\ ELAHE OMIDVAR TEHRANI \\ FERDOWSI UNIVERSITY, IRÁN \\ Received : January 2008. Accepted : June 2010
}

\begin{abstract}
Let $\mathcal{A}$ be an algebra. A sequence $\left\{d_{n}\right\}$ of linear mappings on $\mathcal{A}$ is called a higher derivation if $d_{n}(a b)=\sum_{j=0}^{n} d_{j}(a) d_{n-j}(b)$ for each $a, b \in \mathcal{A}$ and each nonnegative integer $n$. Jewell [Pacific J. Math. 68 (1977), 91-98], showed that a higher derivation from a Banach algebra onto a semisimple Banach algebra is continuous provided that $\operatorname{ker}\left(d_{0}\right) \subseteq \operatorname{ker}\left(d_{m}\right)$, for all $m \geq 1$. In this paper, under a different approach using $C^{*}$-algebraic tools, we prove that each higher derivation $\left\{d_{n}\right\}$ on a $C^{*}$-algebra $\mathcal{A}$ is automatically continuous, provided that it is normal, i. e. $d_{0}$ is the identity mapping on $\mathcal{A}$.
\end{abstract}

Subjclass [2000] : Primary 46L57, 46H40; Secondary 46L05, 47847

Keywords : Derivation, higher derivation, automatic continuity, Sakai theorem. 


\section{Introduction}

Let $\mathcal{A}$ be an algebra. A linear mapping $\delta: \mathcal{A} \rightarrow \mathcal{A}$ is called a derivation if it satisfies the Leibniz rule, i. e. $\delta(a b)=\delta(a) b+a \delta(b)$ for all $a, b \in \mathcal{A}$. If we define the sequence $\left\{d_{n}\right\}$ of linear mappings on $\mathcal{A}$ by $d_{0}=I$ and $d_{n}=\frac{\delta^{n}}{n !}$, where $I$ is the identity mapping on $\mathcal{A}$, then the Leibniz rule ensures us that $d_{n}$ 's satisfy the condition

$$
d_{n}(a b)=\sum_{j=0}^{n} d_{j}(a) d_{n-j}(b)
$$

for each $a, b \in \mathcal{A}$ and each nonnegative integer $n$. This motivates us to consider the sequences $\left\{d_{n}\right\}$ of linear mappings on an algebra $\mathcal{A}$ satisfying (*). Such a sequence is called a higher derivation. Higher derivations were introduced by Hasse and Schmidt [2], and algebraists sometimes call them Hasse-Schmidt derivations. Though, if $\delta: \mathcal{A} \rightarrow \mathcal{A}$ is a derivation then $d_{n}=\frac{\delta^{n}}{n !}$ is a higher derivation, this is not the only example of a higher derivation.

Regarding to a celebrated theorem of Sakai [11, 12], all derivations defined on a $C^{*}$-algebra are automatically continuous. Some results concerning to the theorem are discussed in [8] and [3]. Regarding to the Sakai's theorem we can deduce that the higher derivation $d_{n}=\frac{\delta^{n}}{n !}$ defined on a $C^{*}$-algebra is automatically continuous in the sense that each $d_{n}$ is continuous. This poses the problem of automatic continuity of higher derivations. Many mathematicians could find some affirmative answers to the problem in special cases. Loy [7] proved that if $\mathcal{A}$ is an $(F)$-algebra which is a subalgebra of a Banach algebra $\mathcal{B}$ of power series, then every higher derivation $\left\{d_{n}\right\}: \mathcal{A} \rightarrow \mathcal{B}$ is automatically continuous. Jewell [5], showed that a higher derivation from a Banach algebra onto a semisimple Banach algebra is continuous provided that $\operatorname{ker}\left(d_{0}\right) \subseteq \operatorname{ker}\left(d_{m}\right)$, for all $m \geq 1$. Villena [14], proved that every higher derivation from a unital Banach algebra $\mathcal{A}$ into $\mathcal{A} / \mathcal{P}$, where $\mathcal{P}$ is a primitive ideal of $\mathcal{A}$ with infinite codimension, is continuous. Hejazian and Shatery [4] prove the automatic continuity of higher derivations in the case of $J B^{*}$-algebras.

Here, we prove automatic continuity of higher derivations in the domain of $C^{*}$-algebras. Though, this is a consequence of the Jewell result in [5], our proof just depends on $C^{*}$-algebraic tools. Prior to that, we need some elementary facts concerning higher derivations. For the definition and elementary properties of $C^{*}$-algebras we refer the reader to $[6,9]$ and [10]. One can find a collection of suitable information about automatic 
continuity and some applications of higher derivations in [1] and [13].

\section{Preliminaries}

Let $\mathcal{A}$ be an algebra, $\mathbf{Z}_{k}^{+}=\{0,1, \ldots, k\}$ for $k \in \mathbf{N}$ and $\mathbf{Z}^{+}=\{0,1,2, \ldots\}$. A higher derivation of order $k$ is a sequence $\left\{d_{n}\right\}_{n \in \mathbf{Z}_{k}^{+}}$of linear mappings from $\mathcal{A}$ to $\mathcal{A}$ such that

$$
d_{n}(a b)=\sum_{j=0}^{n} d_{j}(a) d_{n-j}(b)
$$

for all $a, b \in \mathcal{A}$ and $n \in \mathbf{Z}_{k}^{+}$. A sequence $\left\{d_{n}\right\}_{n \in \mathbf{Z}^{+}}$is a higher derivation of infinite order if $\left\{d_{n}\right\}_{n \in \mathbf{Z}_{k}^{+}}$is a higher derivation of order $k$ for each $k \in \mathbf{N}$. A higher derivation $\left\{d_{n}\right\}$ is called normal if $d_{0}=I$ (the identity mapping on $\mathcal{A}$ ). As a simple example, for a derivation $\delta: \mathcal{A} \rightarrow \mathcal{A}$ we can assume the sequence $d_{0}=I, d_{n}=\frac{\delta^{n}}{n !}$. The Leibniz rule implies that $\left\{d_{n}\right\}$ is a higher derivation.

A higher derivation $\left\{d_{n}\right\}$ is called continuous if each $d_{n}$ is continuous. It is said to be onto if $d_{0}$ is onto.

Lemma 2.1. If $\left\{d_{n}\right\}$ is a normal higher derivation on a unital $C^{*}$-algebra with unit $\iota$, then $d_{n}(\iota)=0$ for $n \geq 1$.

Proof. Since $\left\{d_{n}\right\}$ is normal, $d_{1}$ is a dervation and so $d_{1}(\iota)=0$. Let $d_{j}(\iota)=0$ for $1 \leq j \leq n-1$. Then we have

$$
d_{n}(\iota)=d_{n}(\iota . \iota)=\iota . d_{n}(\iota)+\sum_{j=1}^{n-1} d_{j}(\iota) d_{n-j}(\iota)+d_{n}(\iota) . \iota=d_{n}(\iota)+d_{n}(\iota)
$$

Hence $d_{n}(\iota)=0$.

From now on, we assume that $\mathcal{A}$ is a unital $C^{*}$-algebra. In fact, if $\mathcal{A}$ has no identity, we shall consider the $C^{*}$-unitization $\mathcal{A}_{1}$ of $\mathcal{A}$, and define $d_{n}(\iota)=0$ for each $n$.

Recall that if $T$ is a linear mapping and we define $T^{*}$ by $T^{*}(a)=T\left(a^{*}\right)^{*}$ for all $a \in \mathcal{A}$, then $T^{*}$ is a linear mapping on $\mathcal{A}$.

Lemma 2.2. Let $\left\{d_{n}\right\}$ be a higher derivation on a $C^{*}$-algebra $\mathcal{A}$. Then $\left\{d_{n}^{*}\right\}$ is also a higher derivation on $\mathcal{A}$. 
Proof. For each $a, b \in \mathcal{A}$ and $n \in \mathbf{Z}^{+}$we have

$$
\begin{aligned}
& d_{n}^{*}(a b)=\left(d_{n}\left(b^{*} a^{*}\right)\right)^{*}=\left(\sum_{j=0}^{n} d_{j}\left(b^{*}\right) d_{n-j}\left(a^{*}\right)\right)^{*}=\sum_{j=0}^{n} d_{n-j}^{*}(a) d_{j}^{*}(b) \\
= & \sum_{k=0}^{n} d_{k}^{*}(a) d_{n-k}^{*}(b) .
\end{aligned}
$$

Thus $\left\{d_{n}^{*}\right\}$ is a higher derivation.

It is known that the derivation $d: \mathcal{C}^{1}([0,1]) \rightarrow \mathcal{C}([0,1])$ defined by $d(f)=f^{\prime}$ on the dense subalgebra $\mathcal{C}^{1}([0,1])$ of $\mathcal{C}([0,1])$ is not continuous. So the higher derivation $\left\{\frac{d^{n}}{n !}\right\}$ is an example of a discontinuous densely defined normal higher derivation in the $C^{*}$-algebra $\mathcal{C}([0,1])$. In the next section, we will show that this is not the case for everywhere defined higher derivations on $C^{*}$-algebras.

\section{The Result}

Theorem 3.1. Let $\mathcal{A}$ be a unital $C^{*}$-algebra. Then every normal higher derivation $\left\{d_{n}\right\}$ on $\mathcal{A}$ is continuous.

Proof. For each $n \in \mathbf{Z}^{+}$we can write

$$
d_{n}(a b)=\frac{d_{n}^{*}+d_{n}}{2}+i \frac{i d_{n}^{*}-i d_{n}}{2} .
$$

Put $d_{n}^{1}=\frac{d_{n}^{*}+d_{n}}{2}$ and $d_{n}^{2}=\frac{i d_{n}^{*}-i d_{n}}{2}$. Then $d_{n}^{1}$ 's and $d_{n}^{2}$,s are $*$-mappings and $d_{n}^{1}(\iota)=d_{n}^{2}(\iota)=0$ for all $n \in \mathbf{N}$. We also have

$$
\begin{aligned}
& d_{n}^{1}(a b)=a d_{n}^{1}(b)+d_{n}^{1}(a) b+\frac{1}{2} \sum_{j=1}^{n-1} d_{j}(a) d_{n-j}(b)+\frac{1}{2} \sum_{j=1}^{n-1} d_{j}^{*}(a) d_{n-j}^{*}(b), \\
& d_{n}^{2}(a b)=a d_{n}^{2}(b)+d_{n}^{2}(a) b-\frac{i}{2} \sum_{j=1}^{n-1} d_{j}(a) d_{n-j}(b)+\frac{i}{2} \sum_{j=1}^{n-1} d_{j}^{*}(a) d_{n-j}^{*}(b) .
\end{aligned}
$$

It suffices to show that $d_{n}^{1}$ and $d_{n}^{2}$ are continuous for all $n \in \mathbf{Z}^{+}$. At first we prove continuity of $d_{n}^{1}$ 's by induction:

Since $d_{0}^{1}=I, d_{0}^{1}$ is continuous. Suppose that $d_{j}^{1}$ is continuous for $j \leq$ $n-1$. Let $a$ be a self-adjoint element of $\mathcal{A}$ and $\varphi$ be a state on $\mathcal{A}$ such that $|\varphi(a)|=\|a\|$. We may assume that $\varphi(a)=\|a\|$ (If $-\varphi(a)=\|a\|$ then we 
can write $\varphi(-a)=\|-a\|$ and choose the self-adjoint element $-a$ instead of $a)$. Put $\|a\| \iota-a=h^{2}\left(h \geq 0, h \in \mathcal{A}\right.$. Then $\varphi\left(h^{2}\right)=0$ and

$$
\begin{aligned}
& \left|-\varphi\left(d_{n}^{1}(a)\right)-\varphi\left(\frac{1}{2} \sum_{j=1}^{n-1} d_{j}(h) d_{n-j}(h)+\frac{1}{2} \sum_{j=1}^{n-1} d_{j}^{*}(h) d_{n-j}^{*}(h)\right)\right| \\
= & \left|\varphi\left(d_{n}^{1}(\|a\| \iota-a)\right)-\varphi\left(\frac{1}{2} \sum_{j=1}^{n-1} d_{j}(h) d_{n-j}(h)+\frac{1}{2} \sum_{j=1}^{n-1} d_{j}^{*}(h) d_{n-j}^{*}(h)\right)\right| \\
= & \mid \varphi\left(d_{n}^{1}\left(h^{2}\right)-\varphi\left(\frac{1}{2} \sum_{j=1}^{n-1} d_{j}(h) d_{n-j}(h)+\frac{1}{2} \sum_{j=1}^{n-1} d_{j}^{*}(h) d_{n-j}^{*}(h)\right) \mid\right. \\
= & \left|\varphi\left(h d_{n}^{1}(h)\right)+\varphi\left(d_{n}^{1}(h) h\right)\right| \\
\leq & \varphi\left(h^{2}\right)^{1 / 2} \varphi\left(d_{n}^{1}(h)^{2}\right)^{1 / 2}+\varphi\left(d_{n}^{1}(h)^{2}\right)^{1 / 2} \varphi\left(h^{2}\right)^{1 / 2} \\
= & 0 .
\end{aligned}
$$

Hence $\varphi\left(d_{n}^{1}(a)\right)=-\varphi\left(\frac{1}{2} \sum_{j=1}^{n-1} d_{j}(h) d_{n-j}(h)+\frac{1}{2} \sum_{j=1}^{n-1} d_{j}^{*}(h) d_{n-j}^{*}(h)\right)$. Suppose that $\left\{a_{m}\right\}$ is a sequence of self-adjoint elements in $\mathcal{A}$ such that $a_{m} \rightarrow 0$ and $d_{n}^{1}\left(a_{m}\right) \rightarrow b(\neq 0)$. Let $\varphi_{m}$ be a state on $\mathcal{A}$ such that $\left|\varphi_{m}\left(b+a_{m}\right)\right|=\left\|b+a_{m}\right\|$, and let $\varphi_{0}$ be an accumulation point of $\left\{\varphi_{m}\right\}$ in the state space of $\mathcal{A}$. Then we have

$$
\begin{aligned}
\left|\varphi_{m_{k}}\left(b+a_{m_{k}}\right)-\varphi_{0}(b)\right| & =\left|\varphi_{m_{k}}\left(b+a_{m_{k}}\right)-\varphi_{m_{k}}(b)+\varphi_{m_{k}}(b)-\varphi_{0}(b)\right| \\
& \leq\left|\varphi_{m_{k}}\left(b+a_{m_{k}}\right)-\varphi_{m_{k}}(b)\right|+\left|\varphi_{m_{k}}(b)-\varphi_{0}(b)\right| \\
& \leq\left\|b+a_{m_{k}}-b\right\|+\left|\varphi_{m_{k}}(b)-\varphi_{0}(b)\right| \rightarrow 0
\end{aligned}
$$

for some subsequence $\left\{m_{k}\right\}$ of $\{m\}$. Hence $\left|\varphi_{0}(b)\right|=\|b\|$ and so

$$
\varphi_{0}\left(d_{n}^{1}(b)\right)=-\varphi_{0}\left(\frac{1}{2} \sum_{j=1}^{n-1} d_{j}\left(h_{b}\right) d_{n-j}\left(h_{b}\right)+\frac{1}{2} \sum_{j=1}^{n-1} d_{j}^{*}\left(h_{b}\right) d_{n-j}^{*}\left(h_{b}\right)\right),
$$

where $h_{b}=(\|b\| \iota-b)^{1 / 2}$. Similarly one can show that

$$
\left|\varphi_{m_{k}}\left(d_{n}^{1}\left(a_{m_{k}}\right)\right)-\varphi_{0}(b)\right| \rightarrow 0 .
$$

Also if $\left(h_{b+a_{m_{k}}}\right)^{2}=\left\|b+a_{m_{k}}\right\| \iota-\left(b+a_{m_{k}}\right)$ then $h_{b+a_{m_{k}}}^{2} \rightarrow h_{b}^{2}$ and since $h_{b+a_{m_{k}}}$ 's and $h_{b}$ are positive, $h_{b+a_{m_{k}}} \rightarrow h_{b}$. So continuity of $d_{0}^{1}, d_{1}^{1}, \ldots, d_{n-1}^{1}$ implies that

$$
-\varphi_{0}\left(\frac{1}{2} \sum_{j=1}^{n-1} d_{j}\left(h_{b}\right) d_{n-j}\left(h_{b}\right)+\frac{1}{2} \sum_{j=1}^{n-1} d_{j}^{*}\left(h_{b}\right) d_{n-j}^{*}\left(h_{b}\right)\right)
$$




$$
\begin{aligned}
& =\lim _{m_{k} \rightarrow \infty}-\varphi_{m_{k}}\left(\frac{1}{2} \sum_{j=1}^{n-1} d_{j}\left(h_{b+a_{m_{k}}}\right) d_{n-j}\left(h_{b+a_{m_{k}}}\right)\right. \\
& \left.+\frac{1}{2} \sum_{j=1}^{n-1} d_{j}^{*}\left(h_{b+a_{m_{k}}}\right) d_{n-j}^{*} h_{b+a_{m_{k}}}\right) \\
& =\lim _{m_{k} \rightarrow \infty} \varphi_{m_{k}}\left(d_{n}^{1}\left(b+a_{m_{k}}\right)\right) \\
& =\lim _{m_{k} \rightarrow \infty} \varphi_{m_{k}}\left(d_{n}^{1}(b)+d_{n}^{1}\left(a_{m_{k}}\right)\right) \\
& =\varphi_{0}\left(d_{n}^{1}(b)+\varphi_{0}(b)\right) \\
& =-\varphi_{0}\left(\frac{1}{2} \sum_{j=1}^{n-1} d_{j}\left(h_{b}\right) d_{n-j}\left(h_{b}\right)+\frac{1}{2} \sum_{j=1}^{n-1} d_{j}^{*}\left(h_{b}\right) d_{n-j}^{*}\left(h_{b}\right)\right)+\varphi_{0}(b) .
\end{aligned}
$$

Hence $\varphi_{0}(b)=0$, which is a contradiction. So the closed graph theorem guarantees that $d_{n}^{1}$ is continuous.

Similarly we can show that $d_{n}^{2}$ 's are continuous. Whence the continuity of the higher derivation $\left\{d_{n}\right\}$ is deduced.

Acknowledgement. This research was supported by a grant from Ferdowsi University of Mashhad; No. MP89133MIZ. The authors wish to acknowledge the referee for his/her valuable comments and suggestions.

\section{References}

[1] H. G. Dales, Banach Algebras and Automatic Continuity, London Mathematical Society Monographs, New Series, 24. The Clarendon Press, Oxford University Press, Oxford, (2000).

[2] H. Hasse and F. K. Schmidt, Noch eine Begrüdung der theorie der höheren Differential quotienten in einem algebraischen Funtionenkörper einer Unbestimmeten, J. Reine Angew. Math. 177, pp. 215-237, (1937).

[3] S. Hejazian, A.R. Janfada, M. Mirzavaziri and M.S. Moslehian, Achievement of continuity of $(\varphi, \psi)$-derivations without linearity, Bull. Belg. Math. Soc.-Simon Stevn., 14, No. 4, pp. 641-652, (2007).

[4] S. Hejazian, T. L. Shatery, Automatic continuity of higher derivations on J $B^{*}$-algebras, Bull. Iranian Math. Soc., 33, No. 1, pp. 11-23, (2007).

[5] N. P. Jewell, Continuity of module and higher derivations, Pacific J. Math. 68, pp. 91-98, (1977). 
[6] I. Kaplansky, Functional analysis, Some aspects of analysis and probability, Surveys in Applied Mathematics. Vol. 4 John Wiley \& Sons, Inc., New York; Chapman \& Hall, London, (1958).

[7] R. J. Loy, Continuity of higher derivations, Pros. Amer. Math. Soc. 5, pp. 505-510, (1973).

[8] M. Mirzavaziri and M. S. Moslehian, Automatic continuity of $\sigma$ derivations in $C^{*}$-algebras, Proc. Amer. Math. Soc., 134, No. 11, pp. 3319-3327, (2006).

[9] J. G. Murphy, Operator Theory and $C^{*}$-algebras, Academic Press, Inc., Boston, MA, (1990).

[10] T. W. Palmer, Banach algebras and the general theory of $*$-algebras, Vol. I. Algebras and Banach algebras, Encyclopedia of Mathematics and its Applications 49, Cambridge University Press, Cambridge, (1994).

[11] S. Sakai, On a conjecture of Kaplansky, Tohoku Math. J. (2) 12, pp. 31-33, (1960).

[12] S. Sakai, Operator Algebra in Dynamical Systems. Cambridge Univ. press, (1991).

[13] Y. Uchino and T. Satoh, Functional field modular forms and higher derivations, Math. Ann. 311, pp. 439-466, (1998).

[14] A. R. Villena, Lie derivations on Banach algebras, J. Algebra 226, pp. 390-409, (2000).

\section{Shirin Hejazian}

Department of Mathematics

Ferdowsi University

P. O. Box 1159

Mashhad 91775

Iran

e-mail : hejazian@math.um.ac.ir 
Madjid Mirzavaziri

Department of Mathematics

Ferdowsi University

P. O. Box 1159

Mashhad 91775

Iran

e-mail : mirzavaziri@gmail.com

and

\section{Elahe Omidvar Tehrani}

Department of Mathematics

Ferdowsi University

P. O. Box 1159

Mashhad 91775

Iran

e-mail : el_om3@stu-mail.um.ac.ir 